\title{
Cartographie des faciès bio-sédimentaires du Bassin d'Arcachon à partir de l'imagerie FORMOSAT-2
}

\author{
Virginie LAFON ${ }^{1}$, Vincent MARIEU ${ }^{2}$, Rémi BUTEL ${ }^{3}$, Aurélie DEHOUCK ${ }^{4}$, \\ Jean-Marie FROIDEFOND ${ }^{5}$, Gilles TRUT ${ }^{6}$ \\ ${ }^{1}$ UMR CNRS 5805 EPOC, Université Bordeaux 1, \\ Avenue des Facultés, 33405 Talence cedex, France. \\ G.E.O.Transfert, v.lafon@epoc.u-bordeaux1.fr \\ 2 v.marieu@epoc.u-bordeaux1.fr \\ ${ }^{3}$ UFR de Mathématiques et Informatique, Université Bordeaux 1, \\ 351 cours de la Libération, 33405 Talence cedex, France. \\ Remi.Butel@math.u-bordeaux1.fr \\ ${ }^{4}$ a.dehouck@epoc.u-bordeaux1.fr \\ 5 jm.froidefond@epoc.u-bordeauxl.fr
}

${ }^{6}$ IFREMER, Quai du Commandant Silhouette, 33120 Arcachon, France. Gilles.Trut@ifremer.fr

\section{Résumé :}

Cette étude présente les premiers résultats d'un vaste programme de recherche qui ambitionne de définir une méthode de cartographie des platiers intertidaux $d u$ Bassin d'Arcachon (France) par télédétection. Les résultats présentés ici concernent les tests effectués sur l'imagerie Formosat-2 dans le but de discriminer les sédiments et les principales espèces végétales de la lagune. Le résultat d'une classification non supervisée optimisée par la méthode du recuit simulé est ensuite utilisé pour entraîner une classification par minimum de distance. Cette approche permet d'obtenir une carte des faciès bio-sédimentaires dont la précision est estimée à plus de $84 \%$. Cette méthode présente, en outre, un excellent potentiel pour la détermination du pourcentage de couvert végétal des herbiers à zostères.

\section{Abstract :}

This study presents the first results of an ongoing research program that aims to derive thematic maps of the intertidal lagoon of Arcachon (SW Atlantic coast of 
France) from space imagery. At this stage, we present the results of the tests performed on Formosat-2 imagery. Several classification strategies have been tried with the aim to discriminate the sedimentary facies and the main species of the lagoon. The classes calculated by an optimized unsupervised clustering method, based on simulated annealing global minimization technique, were used to train a supervised classification algorithm. This approach allowed us to derive a bio-sedimentary map which overall accuracy is about $84 \%$. Also, this method has the advantage of describing the fraction of vegetation cover of the seagrass.

Mots-clés : Télédétection - Cartographie - Littoral - Classification optimisée Recuit simulé

\section{Introduction}

La dégradation de la qualité des eaux côtières affecte la richesse écologique des écosystèmes littoraux. La gestion et la protection des eaux, notamment des eaux côtières, sont définies, à l'échelle de l'Europe, par la Directive Cadre sur l'Eau (DCE). A l'instar de nombreuses zones littorales, le Bassin d'Arcachon (S.-O. France) fait l'objet d'études pour la mise en œuvre du réseau de référence DCE (DE MONTAUDOUIN, 2007). La flore aquatique, en majorité constituée d'herbiers à Zostera noltii(AUBY \& LABOURG, 1996) occupe plus de la moitié $\mathrm{du}$ domaine (156 $\mathrm{km}^{2}$ au total). Le suivi de ces herbiers est l'un des indicateurs DCE régulièrement actualisé.

La cartographie des herbiers littoraux est fréquemment obtenue par classification de données satellite (ex : FERGUSON \& KORFMACHER, 1997; CALVO et al., 2003; WABNITZ et al., 2008). En 2004, MARIEU et DUPUIS ont montré l'excellent potentiel de l'utilisation d'algorithmes optimisés pour la cartographie thématique du Bassin d'Arcachon à partir de données Spot, des tests complémentaires, centrés sur la cartographie de la végétation halophile, ainsi qu'une validation, sont proposés dans le cadre des présents travaux.

Nous présentons ici une stratégie de cartographie du Bassin d'Arcachon par imagerie Formosat-2 (F2), capteur THR décrit dans la seconde partie de cette étude. Pour élaborer cette stratégie, nous avons testé, à partir des canaux F2, différents algorithmes de classification non supervisée pour générer des zones d'entraînement clairement distinctes sur le plan spectral. Nous souhaitons discriminer le sable, la vase, les herbiers (en fonction du pourcentage de couvert) et le pré salé (ou schorre) dont seules les spartines seront ici discriminées, le reste du schorre étant traité comme une simple entité. Dans le cadre de ces travaux, nous n'avons pas considéré le cas des dépôts d'algues qui peuvent être confondus 
avec l'herbier. Ils peuvent toutefois couvrir des surfaces importantes (15 à $20 \mathrm{~km}^{2}$ sur les estrans pour l'espèce Monostroma d'après AUBY et al., 1994). Nous avons utilisé des algorithmes rapides convergeant vers des minima locaux et un algorithme plus performant, mais plus lent, convergeant vers un minimum global, le recuit simulé, déjà appliqué à l'analyse de bases de données (ex: BUTEL et al., 2002). Ces méthodes sont décrites dans la troisième partie de cette étude. La quatrième partie synthétise les résultats de ces tests ainsi que la validation de la carte bio-sédimentaire issue de la classification supervisée de l'imagerie F2 basée sur les parcelles d'entraînement définies par classification non supervisée.

\section{Moyens d'observation}

\subsection{Imagerie FORMOSAT-2}

La scène F2 utilisée dans le cadre de cette étude a été acquise le 27 Août 2007 à basse mer. La scène a une résolution spatiale de $2 \mathrm{~m}$ et comporte 4 bandes spectrales (B1 à B4) dans les longueurs d'onde bleu $(0,45-0,52 \mu \mathrm{m})$, vert $(0,52-$ $0,60 \mu \mathrm{m})$, rouge $(0,63-0,69 \mu \mathrm{m})$ et proche infrarouge $(0,76-0,90 \mu \mathrm{m})$. La scène a été géoréférencée à l'aide de 27 points d'appui régulièrement distribués autour du Bassin et projetée dans le système Lambert 3. Après application du modèle de correction géométrique, ces points d'appui sont localisés avec une précision moyenne (RMS) inférieure à $0,5 \mathrm{~m}$.

\subsection{Photographies obliques}

Une mission de photographie a été réalisée à bord d'un ULM le 30 août 2007 à marée basse. Vingt-sept enregistrements obliques $\left(5 \mathrm{~km}^{2}\right)$ ont été effectués au sud du Bassin d'Arcachon à l'aide d'un appareil numérique Sony Cyber-Shot. Ces observations ont été utilisées pour l'identification des spartines et du schorre.

\subsection{Observations in situ}

Des pointages GPS ont été recueillis entre 2003 et 2008 représentatifs des différents types de sédiments, mais également des herbiers et du schorre. Pour l'herbier, sujet à de profondes modifications saisonnières à interannuelles, seules les observations effectuées en septembre 2007 dans le cadre de la cartographie DCE suivant les recommandations mises en œuvre dans le cadre du réseau de surveillance des biocénoses benthiques ont été intégrées à cette étude. Ces levés, localisés à l'aide d'un DGPS sub-métrique GeoXT Trimble, comportent trois classes de pourcentage de couverture végétale : 0 à $25 \%, 25$ à $75 \%$ et 75 à $100 \%$. 


\subsection{Spectroradiométrie}

Les mesures spectroradiométriques réalisées entre 1994 et 2005 (LAFON \& FROIDEFOND, 1997 ; MARIEU \& DUPUIS, 2004), ainsi qu'une série de mesures réalisée en 2007 dans le cadre du suivi surfacique DCE, sont exploitées ici. Depuis 2003, deux spectroradiomètres TRIOS (RAMSES-ACC et RAMSESARC reliés par une interface TRIOS IPS104) sont mis en œuvre pour mesurer la luminance des cibles $\left(\mathrm{L}_{\mathrm{u}}\right)$ et l'éclairement de la voûte céleste $\left(\mathrm{E}_{\mathrm{d}}\right)$. Avant cela, un Spectron SE-590 mesurait $\mathrm{L}_{\mathrm{u}}$ et $\mathrm{L}_{\mathrm{d}}$ (luminance incidente). L'ensemble de ces spectres a été traité afin de restituer pour chaque cible sa réflectance de télédétection $\left(\mathrm{R}_{\mathrm{RS}}=\mathrm{L}_{\mathrm{u}} / \pi \mathrm{L}_{\mathrm{d}}=\mathrm{L}_{\mathrm{u}} / \mathrm{E}_{\mathrm{d}}\right)$. Puis, les spectres équivalents $\mathrm{F} 2$ ont été reconstruits en tenant compte de la sensibilité spectrale du capteur du satellite. La bibliothèque de spectres est constituée de 130 enregistrements pour moitié accompagnés de mesures du taux d'humidité, de la granulométrie et de la biomasse. La présence de biofilms lors des acquisitions est également indiquée.

\section{Méthodes de classification et cartographie thématique}

Le déroulement des tests de classification ainsi que la stratégie générale suivie pour obtenir une carte bio-sédimentaire détaillée du Bassin d'Arcachon basée sur l'imagerie F2 est décrite par la figure 1. La scène a d'une part été calibrée à l'aide de 23 spectres radiométriques d'objets sédimentaires et végétaux mesurés in situ. Simultanément, une imagette (93624 pixels) a été extraite sur une zone bien documentée. Puis les différentes méthodes de classification non supervisée ont été appliquées à cette imagette.

Kmeans (MACQUEEN, 1967) et Isodata sont des méthodes qui visent à minimiser l'inertie intra-classe (somme des distances normalisées au centre de gravité de la classe pour chaque canal). Isodata introduit des paramètres qui lui permettent progressivement de tester la similarité intra- et inter-classes jusqu'à parfois séparer ou fusionner des classes.

Les trois autres méthodes utilisent une fonction d'optimisation. Celle-ci fait des appels à une fonction de calcul de la somme de la distance de chaque pixel au centre de gravité le plus proche jusqu'à trouver la valeur minimale. Les méthodes de Powell et d'Amoeba s'avèrent très rapides mais peuvent converger vers un minimum local. La méthode du recuit simulé (KILPATRICK et al., 1983) tire au hasard (Monte Carlo) une proposition de solution, calcule la valeur de la fonction à minimiser, et, en fonction du minimum local courant, choisit (i) de continuer dans la même direction de recherche si la nouvelle valeur est inférieure, (ii) de tirer au hasard une probabilité de sortir du voisinage si la nouvelle valeur est supérieure au mimimum local courant. Cette probabilité est fonction d'un 
paramètre appelé température (INBERG, 1993) qui décroît au fur et à mesure que le temps (nombre d'itérations) augmente de façon à échapper à des minima locaux médiocres en début de calcul (et sur lesquels les autres méthodes de minimisation peuvent s'arrêter). On ne peut prouver que cette méthode converge toujours vers la bonne solution, mais elle est à priori plus performante. Le recuit simulé est très gourmand en temps de calcul, il ne peut être appliqué à la scène entière (près de 40 millions de pixels).

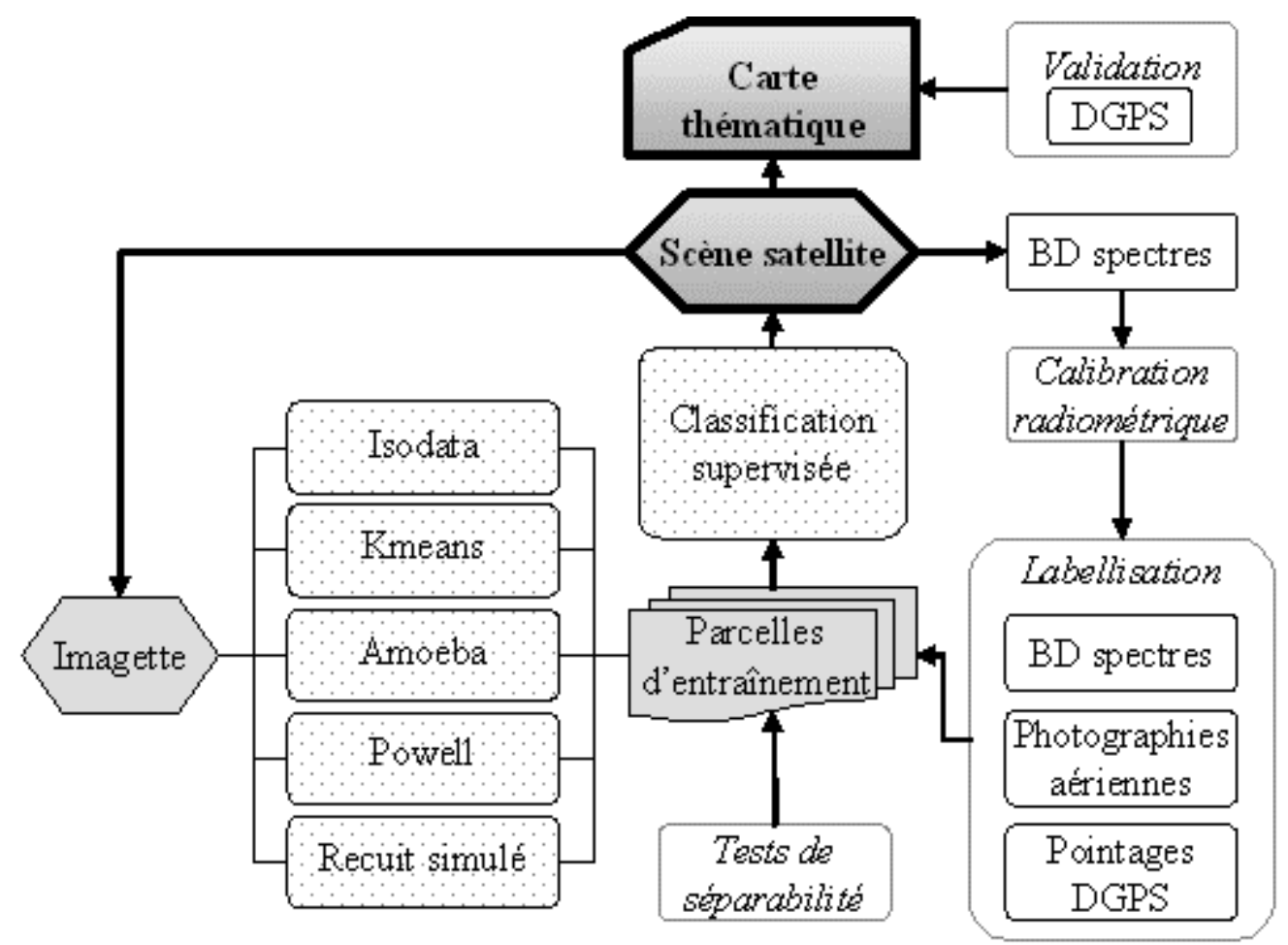

Figure 1. Méthode de traitement de la scène Formosat-2

Ces méthodes permettent de définir un jeu de parcelles d'entraînement dont la séparabilité est analysée par la méthode Jeffries-Matusita. Enfin les parcelles d'entraînement sont labellisées puis transférées à un algorithme de classification supervisée. La carte résultante est validée à l'aide d'observations in situ. Ces traitements ont été exécutés sous ENVI 4.1 (® RSI).

\section{Résultats et discussion}

\subsection{Calibration de la scène}

La calibration de la scène F2 à partir des spectres in situ de référence est satisfaisante $\left(\mathrm{r}^{2}\right.$ de 0,$85 ; 0,84 ; 0,86 ; 0,80$, pour les bandes $\mathrm{B} 1$ à $\left.\mathrm{B} 4\right)$. Elle permet de calculer les réflectances $\mathrm{F} 2$ pour les comparer avec la bibliothèque de spectres. 


\subsection{Classification}

Les méthodes Powell et Amoeba donnent les résultats les plus médiocres avec une classe regroupant des zones de spartines et de schorre. Les herbiers sont représentés par seulement deux classes. Ces parcelles définissent donc mal les différents écosystèmes de la lagune.

Les méthodes Isodata et Kmeans, quoique plus adaptées notamment pour décrire l'herbier, ne permettent pas de discriminer le schorre des spartines. En outre, l'herbier très dense et le schorre apparaissent dans une classe unique. Enfin les classes représentatives de la vase et de très faible couvert d'herbier ne sont pas séparables sur le plan spectral. Ces parcelles ne sont donc pas encore suffisamment représentatives, en particulier dans le contexte DCE.

Le recuit simulé offre 19 classes bien toutes séparables sur le plan spectral. Ces classes ont été labellisées. Deux d'entres elles représentent l'eau, l'une d'elle le schorre, une autre les spartines, deux classes décrivent le sable de plage, cinq le sable des platiers (en fonction de la teneur en eau), trois classes la vase (dont une classe qui montre la présence de biofilms) et enfin cinq classes l'herbier (dont 2 pour lesquelles le taux de couverture est connu). Afin de mieux prendre en compte la variabilité du couvert, 11 classes complémentaires constituées de petites surfaces de réflectance homogène ont été ajoutées à ces 19 classes principales. Celles-ci sont centrées sur deux espèces de spartines et une zone de schorre absentes de l'imagette ainsi que six zones d'herbiers situés en milieu très humide et pour lesquels le pourcentage de couvert est connu. Enfin, deux classes ont été ajoutées pour représenter l'eau profonde et les zones faiblement immergées. Les 30 parcelles résultantes sont parfaitement distinctes sur le plan spectral. Une carte a été générée à l'aide de ces 30 parcelles (figure 2). Par soucis de clarté, la figure 2 comporte 8 couleurs, un zoom plus détaillé est également présenté.

\subsection{Validation de la carte bio-sédimentaire}

La validation de la carte a été effectuée à l'aide de 154 observations in situ. La précision globale de la carte (overall accuracy d'après FOODY, 2002) est de plus de $84 \%$, c'est-à-dire très proche des $85 \%$ préconisés par THOMLINSON et al. (1999). En outre, ce score est meilleur que celui obtenu pour la cartographie d'herbiers (68\% en moyenne sur 14 sites) dans les Caraïbes (WABNITS et al. 2008). Pour cette validation, nous n'avons pas regroupé le sable et la vase, car seulement 4 observations permettent de valider la classe «vase». Ces quatre observations sont toutefois correctement classées. Nous notons également que 4 
points GPS «sable» sont classés «vase », mettant en évidence quelques difficultés de discrimination entre les faciès sédimentaires.

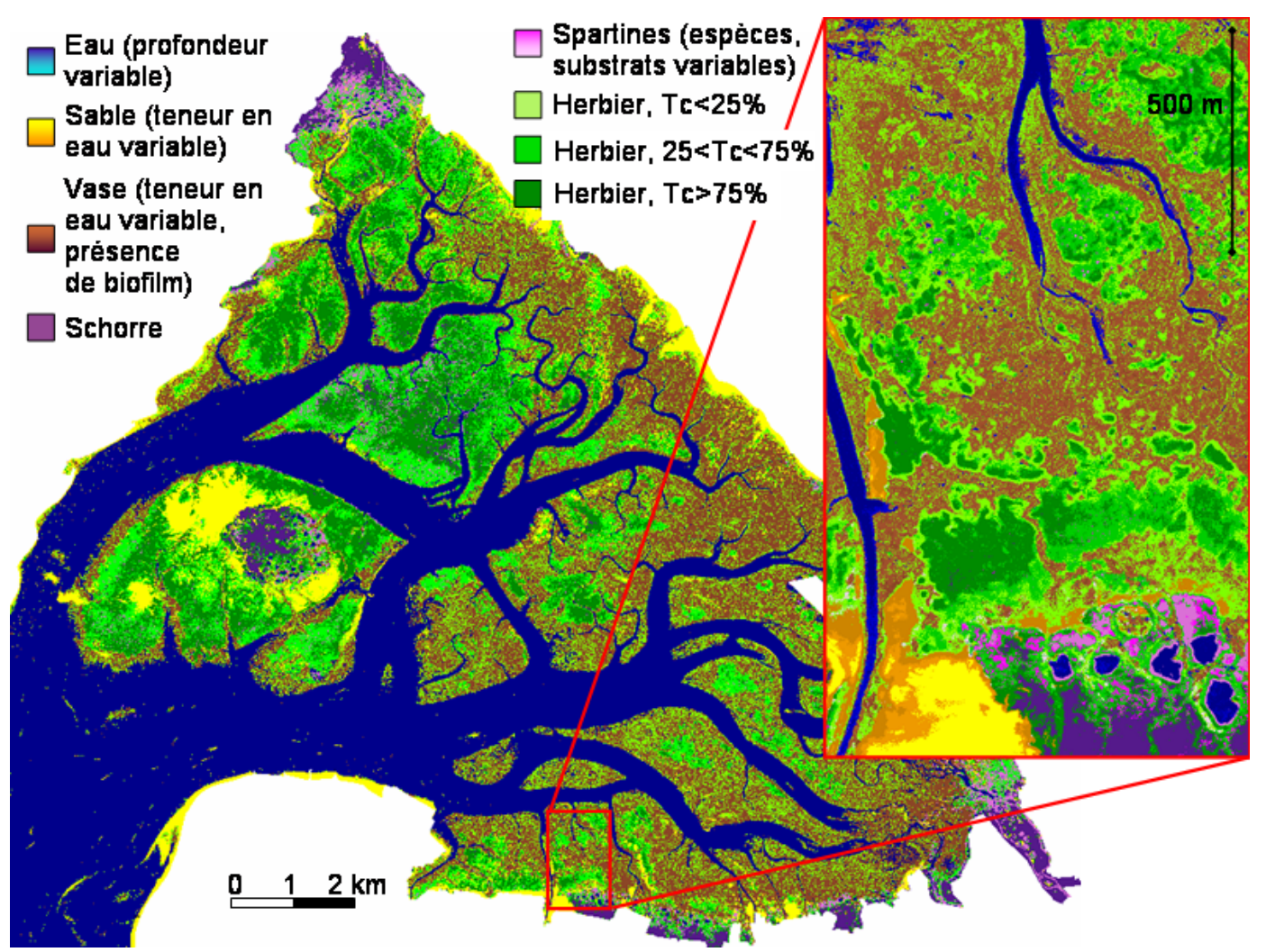

Figure 2. Carte bio-sédimentaire du Bassin d'Arcachon (Août 2007). Tc

représente le pourcentage de couverture végétale

Du point de vue de la procédure (voir matrice de confusion tableau 1), la principale difficulté réside dans la discrimination correcte du schorre confondu avec l'herbier ou avec des spartines. Du point de vue de l'utilisateur, l'ensemble des faciès est très correctement représenté (précision toujours supérieure aux 70\% préconisés par THOMLINSON et al., 1999). Toutefois, des zones classées « sédiments nus » correspondent à l'herbier ou aux spartines. Dans le cas des spartines, ce sont des zones où les spartines ont une extension spatiale de l'ordre $\mathrm{du}$ pixel. Une analyse de la composition spectrale intra-pixel permettrait de résoudre ces erreurs. On peut aussi supposer que les spartines sur des zones sableuses sont desséchées en fin d'été et ont un spectre plus proche de celui d'un minéral que d'un végétal, elles peuvent dans ce cas être mal classées. De plus, on note certaines zones où l'herbier, très dense, est classé «schorre» (confusion expliquée par la séparabilité moindre d'une des classes herbier et de la classe schorre), et d'autres, plutôt aux abords des chenaux, où l'herbier est assimilé à des 
spartines. Environ 0,2\% des pixels des platiers intertidaux sont classés « schorre » et $0,7 \%$ sont classés «spartines ». Ces erreurs ont donc un poids relativement faible.

Tableau 1. Matrice de confusion ou matrice d'erreur de la classification.

\begin{tabular}{|c|c|c|c|c|c|c|c|}
\hline \multicolumn{8}{|c|}{ Observations } \\
\hline \multirow{7}{*}{$\begin{array}{l}\text { Pixels } \\
\text { classés }\end{array}$} & & $\begin{array}{c}\text { Sédiment } \\
\mathrm{nu}\end{array}$ & Herbier & Spartine & Schorre & Total & $\begin{array}{c}\text { \% Précision } \\
\text { utilisateur }\end{array}$ \\
\hline & Sédiment nu & 27 & 5 & 5 & - & 37 & 73 \\
\hline & Herbier & - & 60 & 7 & 3 & 70 & 86 \\
\hline & Spartine & - & - & 29 & 4 & 33 & 88 \\
\hline & Schorre & - & - & - & 14 & 14 & 100 \\
\hline & Total & 27 & 65 & 41 & 21 & 154 & \\
\hline & $\begin{array}{l}\text { \% Précision } \\
\text { Procédure }\end{array}$ & 100 & 92 & 71 & 67 & & \\
\hline
\end{tabular}

\subsection{Estimation du taux de couvert par classification}

Cinq des onze classes d'herbier sont labellisées en fonction du pourcentage de couverture végétale. Les classes restantes ont été labellisées par comparaison avec les spectres moyens des classes déjà labellisées doublée de l'analyse de séparabilité interclasses, qui permet de déterminer les classes les plus semblables sur le plan spectral. Nous avons finalement comparé la densité F2 avec 49 estimations in situ du taux de couverture (différentes des observations utilisées lors de la phase de labellisation). La précision obtenue est de $59 \%$, la perception du pourcentage de surface couvert par l'herbier étant plutôt pessimiste. Les pixels présentant une sous-estimation du taux de couvert se situent généralement dans des confluences de chenaux ou à proximité des principaux chenaux indiquant. Les terrains y sont vraisemblablement inondés sous l'effet de la marée. Il en résulte une atténuation des réflectances analysée comme un plus faible pourcentage de couverture végétale.

\section{Conclusions et perspectives}

Ces tests montrent l'efficacité d'une double approche de classification pour la cartographie du Bassin d'Arcachon. La première étape repose sur un algorithme de classification non supervisé optimisé par la méthode du recuit simulé qui permet une discrimination optimale des faciès et espèces végétales présents dans le milieu. Cette méthode étant très lourde à mettre en oeuvre, elle est appliquée à une 
petite surface du domaine. Les classes obtenues sont labellisées, notamment à l'aide d'une bibliothèque de spectres in situ auxquels sont comparés les moyennes radiométriques des classes issues de la classification. Afin de mieux représenter la variété bio-sédimentaire du domaine, et en particulier, dans notre cas, d'associer des classes à des pourcentages de couvert déterminés en des lieux très dispersés de la lagune, des petites zones de réflectance homogène ont été adjointes aux classes initiales. L'ensemble de ces parcelles d'entraînement a finalement permis de lancer une classification supervisée par minimum de distance. La précision de la carte obtenue, estimée à l'aide de 154 observations, est de plus de $84 \%$. Tant du point de vue de la procédure que de celui de l'utilisateur, l'herbier, habitat particulièrement ciblé dans le cadre du suivi surfacique DCE, est correctement cartographié, à l'exception des zones où les dépôts d'algues vertes pourraient être confondus avec l'herbier. En outre, le pourcentage de la surface couverte par l'herbier peut être estimé. La précision du taux de couvert est de $59 \%$ pour trois classes $(0-25 \%, 25-75 \%, 75-100 \%)$. Cette précision pourrait vraisemblablement être améliorée en acquérant une image plus proche de la basse mer, en évitant ainsi la saturation des sols qui influence l'estimation du pourcentage de couvert de l'herbier. Enfin, si cette étude montre qu'il est possible de discriminer le schorre des spartines, des expérimentations en cours montrent que le schorre présente une réponse spectrale en étage, cohérente avec des assemblages d'espèces observés sur le terrain. Ces analyses seront poursuivies afin de définir les possibilités de cartographie du schorre par télédétection très haute résolution.

\section{6. $\quad$ Remerciements}

Nous remercions Spot Image et le NSPO, pour le geste commercial qui a facilité l'utilisation des données issues du satellite Formosat-2. Les estimations du taux de couvert de l'herbier ont été effectuées par F. d'Amico, I. Auby, C. Cantin, J.P. Dreno, M. Duval, N. Masson-Neaud, D. Maurer, M. Plus, M.P. Tournaire et G. Trut, de la station marine Ifremer d'Arcachon. Merci finalement à Ifremer et l'Agence de l'Eau Adour Garonne qui cofinancent le suivi surfacique DCE.

\section{Références bibliographiques}

AUBY I., MANAUD F., MAUREER D., TRUT G. (1994). Etude de la prolifération des algues vertes dans le bassin d'Arcachon. Rapport d'étude IFREMER - CEMAGREF - SSA - SABARC, 270 p.

AUBY I., LABOURG J.P. (1996). Seasonal dynamics of Zostera Noltii Hornem. in the bay of Arcachon (France). Journal of Sea Research, vol. 35, $\mathrm{n}^{\circ} 4$, pp. 269-277. 
BUTEL R., DUPUIS H., BONNETON P. (2002). Spatial Variability of Wave Conditions on the French Atlantic Coast using In-Situ Data. Journal of Coastal Research, vol. SI 36, pp. 96-108.

CALVO S., CIRAOlO G., LA LOGGIA G. (2003). Monitoring Posidonia oceanica meadows in a Mediterranean coastal lagoon (Stagnone, Italy) by means of neural network and ISODATA classification methods. International Journal of Remote Sensing, vol. 24, pp. 2703-2716.

DE MONTAUDOUIN X. (2007). Evaluation de la qualité biologique des milieux littoraux semi-fermés. Programme LITEAU 2, Rapport intermédiaire, Université Bordeaux 1, 37 p.

FERGUSON R. L., KORFMACHER K. (1997). Remote sensing and GIS analysis of seagrass. Aquatic Botany, vol. 58, pp. 241-258.

FOODY, G. M. (2002). Status of land cover classification accuracy assessment. Remote Sensing of Environment, vol. 80, pp. 185 - 201.

INBERG, L. (1993). Simulated Annealing: Practice versus Theory. Journal of Mathematical Computer Modelling, vol.18, $\mathrm{n}^{\circ} 11$, pp. 29-57.

KILPATRICK S., GELATT C. D., VECCHI M. P. (1983). Optimization by Simulated Annealing. Science, vol. 220, n 4598, pp. 671-680.

LAFON V., FROIDEFOND J. M. (1997). Cartographie bio-sédimentaire d'une lagune à marée basse à partir de données SPOT. Exemple du bassin d'Arcachon, France. Bulletin de la Société Française de Photogrammétrie et Télédétection, $\mathrm{n}^{\circ} 147$, pp. 13-24.

MACQUEEN J. (1967). Some methods for classification and analysis of multivariate observations. In: L. M. Le Cam \& J. Neyman [eds.], Proceedings of the fifth Berkeley symposium on mathematical statistics and probability, vol. 1. University of California Press, Berkeley, pp. 281-297.

MARIEU V., DUPUIS H. (2004). Evaluation du potentiel de l'imagerie SPOT5 dans le secteur du bassin d'Arcachon. Cartographie du milieu lagunaire. Rapport de fin de contrat du suivi du littoral par SPOT5: Appel à proposition CNESIFEN.

THOMLINSON J. R., BOLSTAD P. V., COHEN W. B. (1999). Coordinating methodologies for scaling landcover classifications from site-specific to global: steps toward validating global map products. Remote Sensing of Environment, vol. 70, pp. 16-28.

WABNITZ C. C., ANDREFOUËT S., TORRES-PULliZA D., MÜLLERKARGER F. E., KRAMER P.A. (2008). Regional-scale seagrass habitat mapping in the wider Caribbean region using Landsat sensors: Applications to conservation and ecology. Remote Sensing of Environment, vol. 112, pp. 3255-3497. 